\title{
Effect of gyromagnetic fields on human prostatic adenocarcinoma cells
}

\author{
This article was published in the following Dove Press journal: \\ OncoTargets and Therapy \\ 24 November 2015 \\ Number of times this article has been viewed
}

\author{
Hongen Lei' \\ Yongde $\mathrm{Xu}{ }^{\prime}$ \\ Ruili Guan' \\ Meng $\mathrm{Li}^{2}$ \\ $\mathrm{Yu} \mathrm{Hui}{ }^{3}$ \\ Zhezhu Gao' \\ Bicheng Yang' \\ Zhongcheng Xin' \\ 'Andrology Center, Peking University \\ First Hospital, Peking University, \\ Beijing, ${ }^{2}$ Department of Urology, \\ General Hospital of Ningxia Medical \\ University, Yinchuan, ${ }^{3}$ Department of \\ Urology, The First Affiliated Hospital \\ of Soochow University, Soochow, \\ People's Republic of China
}

Correspondence: Zhongcheng Xin Andrology Center, Peking University First Hospital, Peking University, No 8, Xishiku Street, Xicheng District, Beijing 100034, People's Republic of China

Tel +861083228989

Fax +861083222822

Email xinzc@bjmu.edu.cn
Purpose: To investigate the biological effect of gyromagnetic fields (GMFs) on cell proliferation and apoptosis of human prostatic adenocarcinoma cells and explore the underlying mechanisms. Methods: PC-3 cells were grouped into normal control (NC) and GMF treatment groups. Cell proliferation was analyzed with kit- 8 and Ki67 immunofluorescence staining, while cell apoptosis was analyzed with flow cytometry double staining of Annexin V-PE/7-AAD. The Akt and p38 MAPK/Caspase signaling pathways were analyzed by western blotting and immunofluorescence staining, and cell polarization was analyzed with PARD3.

Results: Cell proliferation and activity of the Akt pathway were significantly decreased by the GMF, while cell apoptosis, activity of p38 MAPK, and PARD3-positive cell number were significantly increased in the GMF group compared to the NC group.

Conclusion: GMFs inhibit cell proliferation, induce apoptosis, and regulate tumor cell polarity conditions, potentially through down-regulating Akt, activating the p38 MAPK/Caspase pathway, and promoting PARD3 expression in PC-3 cells.

Keywords: apoptosis, gyromagnetic fields, PC-3 cells, prostate cancer

\section{Introduction}

Prostate cancer is the most common cancer in elderly men $>70$ years of age, especially in developed countries. Its incidence is 85.6 (per 100,000 men per year, age-standardized rate) in the USA, compared with 7.2 in Asia, while there has been a trend of increasing incidence in developing countries in recent years. ${ }^{1}$ Older age, a positive family history, and black race are the three most common risk factors for prostate cancer. ${ }^{2}$ Men at elevated risk for prostate cancer should undergo early prostatespecific antigen (PSA) testing, ${ }^{3}$ though there is no strong evidence that prostate-specific antigen screening could reduce mortality due to prostate cancer. ${ }^{4}$ Current treatment options are available for patients with different clinical grades and stages, including active surveillance and watchful waiting, radical prostatectomy, definitive radiotherapy, high-intensity focused ultrasound, cryosurgery, and hormonal therapy. ${ }^{5-7}$ However, there are complications related to treatment of prostate cancer, and this disease is doomed to progress to the final stages of metastatic androgen-independent prostate cancer with its attendant substantial threat of mortality. ${ }^{8}$

Recently, convergence of the life sciences, physical sciences, and engineering has brought the biomedicine world into a new era. ${ }^{9}$ Therapeutic magnetic fields, good paradigms of convergence medicine, have been demonstrated to have beneficial results on benign prostatic hyperplasia, ${ }^{10,11}$ human breast carcinoma, ${ }^{12}$ peripheral nerve regeneration, ${ }^{13}$ hand osteoarthritis, ${ }^{14,15}$ osteonecrosis,${ }^{16}$ and nucleic acid delivery. ${ }^{17}$ Magnetic fields have been proven to be safe, non-invasive methods for the modification of numerous cells and tissues, especially in the musculoskeletal system. ${ }^{18}$ It is interesting 
to note that magnetic therapy can cause a significant reduction in prostatic volume with no side effects in a canine model ${ }^{10}$ and a significant improvement in clinical symptoms in benign prostatic hyperplasia patients. ${ }^{11}$ Besides, magnetic fields induce a decrease in cell growth in human breast carcinoma cells; however, the mechanism of magnetic fields on cell growth is still not clear. ${ }^{12}$ Gyromagnetic fields (GMFs) are a novel type of magnetic field with its field strength varying with time and obeying the distribution regularity of a sine curve. Here, to test if GMFs could be a novel method in the treatment of prostate cancer, we investigated its effect on cell growth and apoptosis in human prostatic adenocarcinoma PC-3 cells.

\section{Materials and methods Cell culture}

Human prostatic adenocarcinoma cell line PC-3 was obtained from the American Type Culture Collection (ATCC, Manassas, VA, USA). Cells were cultured at $37^{\circ} \mathrm{C}$ in a $5 \% \mathrm{CO}_{2}$ atmosphere in a humidified incubator. F-12K culture medium (Thermo Fisher Scientific, Waltham, MA, USA) was used, supplemented with $10 \%$ fetal bovine serum and $2 \mathrm{mM}$ of L-glutamin. All experimental protocols in this study were approved by the Ethical Committee of Peking University First Hospital.

\section{Gyromagnetic fields treatments}

PC-3 experimental groups were exposed to GMFs by a CKJ-II (QLX-II) Gyromagnetic Therapy Machine (Beijing Gyromagnetic Medical Equipment Co., Ltd., Beijing, People's Republic of China). The work parameters were the GMF strength varied with time in the target area and obeyed the distribution regularity of a sine curve; the rotating frequency of the GMF was $6 \mathrm{~Hz}$; the maximum field strength was $300 \mathrm{mT}$; and the magnetic flux density was $8.3 \mathrm{mT} / \mathrm{Hz}^{2}$ (Figure 1). The top and bottom surfaces of the two magnetic poles were at a distance of $32 \mathrm{~cm}$. Cell samples were placed in the gyromagnetic therapeutic machine according to the manufacturer's instructions.

\section{Cell counting test}

The cell proliferation assay was performed using a cell counting kit-8 (CCK-8, Dojindo Laboratories, Kumamoto, Japan) according to the manufacturer's instructions. Fivehundred cells were seeded into 96 wells and cultured in the F-12K complete medium. At 24 hours after GMF treatment according to the above mentioned method, $10 \mu \mathrm{L}$ of CCK-8 solution was added into the 96 wells to make the working solution and incubated for 1 hour at $37^{\circ} \mathrm{C}$. Absorbance at $450 \mathrm{~nm}$ was measured to calculate cell numbers according to optical density values.

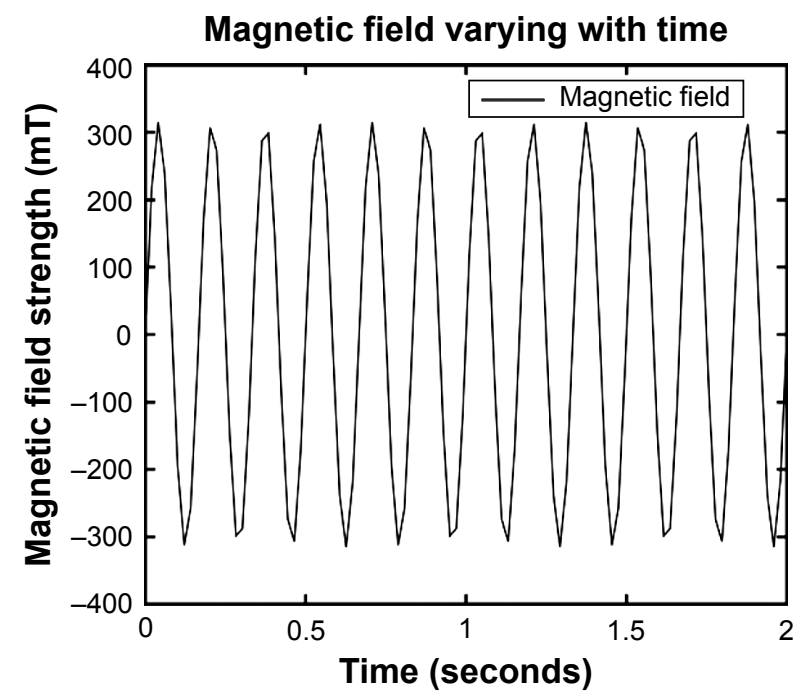

Figure I Gyromagnetic field strength distribution varying with time using a CKJ-II (QLX-II) Gyromagnetic Therapy Machine.

Notes: Gyromagnetic field strength varied with time in the target area and obeyed the distribution regularity of a sine curve.The rotating frequency of the gyromagnetic field was $6 \mathrm{~Hz}$, the maximum field strength was $300 \mathrm{mT}$, and the magnetic flux density was $8.3 \mathrm{mT} / \mathrm{Hz}^{2}$.

\section{Immunofluorescence}

Twenty-four hours after treatment with a GMF according to the above mentioned method, cells were fixed with cold $4 \%$ formaldehyde for 10 minutes at room temperature and subsequently incubated with phosphate-buffered saline (PBS) containing $0.3 \%$ triton X-100 and $2 \%$ bovine serum albumin for 30 minutes at room temperature. This was followed by incubation with primary antibodies against Ki67 (Abcam, Cambridge, MA, USA), Cleaved Caspase-3 (Cell Signaling Technology, Danvers, MA, USA), and PARD3 (Abcam) overnight at $4^{\circ} \mathrm{C}$. After rinsing with PBS, cells were incubated with Alexa594-conjugated goat anti-rabbit IgG antibody. After a further rinse with PBS, the cells were incubated with $1 \mu \mathrm{g} / \mathrm{mL}$ of $4^{\prime}, 6$-diamidino-2-phenylindole (Sigma-Aldrich, St Louis, MO, USA) for nuclear staining.

\section{Flow cytometry}

The cell flow cytometry assay was performed using an Annexin V-PE/7-AAD Apoptosis Detection Kit (KeyGEN BioTECH, Nanjing, People's Republic of China) according to the manufacturer's instructions. Cells were seeded into six wells and cultured in the F-12K complete medium. Twentyfour hours after treatment with a GMF as mentioned above, cells were collected and resuspended in $500 \mu \mathrm{L}$ of binding buffer, after which $5 \mu \mathrm{L}$ of 7-AAD solution and $1 \mu \mathrm{L}$ of Annexin V-PE solution were added; cells were then incubated for 15 minutes in the dark. Finally, cells were analyzed by flow cytometry (BD influx, BD Biosciences, Franklin Lakes, NJ, USA) within 1 hour. 


\section{Western blot analysis}

Whole-cell protein samples were prepared by homogenization in RIPA Lysis Buffer (KeyGEN BioTECH) supplemented with a protease and phosphatase inhibitor cocktail. Cell lysates containing $20 \mu \mathrm{g}$ of protein were electrophoresed in sodium dodecyl sulfate polyacrylamide gel electrophoresis and then transferred to a polyvinylidene fluoride membrane (EMD Millipore, Billerica, MA, USA). The membrane was blocked with 5\% skimmed milk for 1 hour at room temperature and incubated overnight at $4^{\circ} \mathrm{C}$ with primary antibodies against phospho-Akt (Cell Signaling Technology), Akt (Cell Signaling Technology), phospho-p38 mitogen-activated protein kinases (MAPK) (Cell Signaling Technology), p38 MAPK (Cell Signaling Technology), Cleaved Caspase-9 (Cell Signaling Technology), and GAPDH (CWBIO, Beijing, People's Republic of China). After the hybridization of secondary antibodies for 1 hour at room temperature, detection was achieved by acquiring the image directly in a chemiluminescencecompatible digital imaging system (C-DiGit Blot Scanner, LI-COR Biosciences, Cambridge, UK).

\section{Image analysis and quantification}

Images of cells were captured using a digital camera (Leica DM2500, Leica Microsystems, Wetzlar, Germany). They were then imported into Image-Pro plus software (version 6.0, Media Cybernetics Inc, Bethesda, MD, USA) for calculating the mean density and dot numbers of each sample. The cell apoptosis rates of the flow cytometry assay were analyzed with FlowJO software (version 7.6.2, Tree Star Inc., Ashland, OR, USA). The integrated density value of each protein band of the western blot images was analyzed with ImageJ software (version 1.46r, ImageJ, NIH, Bethesda, MD, USA).

\section{Statistical analysis}

The data are presented as means \pm SD. One-way analysis of variance followed by the Student-Newman-Keuls test was used to evaluate whether differences among groups were significant. All calculations were performed using SPSS statistical software (version 13.0, SPSS, Chicago, IL, USA). $P<0.05$ was considered significant.

\section{Results}

\section{Gyromagnetic fields inhibited cell proliferation in PC-3 cells}

To investigate the effects of GMFs on cell proliferation in PC-3 cells, a cell-counting assay was performed 24 hours after GMF treatment. Cell proliferation was significantly inhibited when compared with the normal control (NC) group $(P<0.05)$, especially in the GMF 10 -minute group (Figure 2A). We then checked cell numbers in the GMF 10 minutes versus the NC group for 4 days; cell growth was significantly inhibited in the GMF 10-minute group $(P<0.05$; Figure 2B).

Ki67 was strongly associated with cell proliferation and growth; immunofluorescence staining in PC-3 cells showed that the Ki67-positive cell number was significantly decreased in the GMF 10-minute group $(P<0.05) 24$ hours after treatment with a GMF (Figure $2 \mathrm{C}$ and $\mathrm{D}$ ). These data suggested that GMFs could inhibit cell proliferation in PC-3 cells.

\section{Gyromagnetic fields suppressed Akt signaling in PC-3 cells}

To demonstrate the molecular basis of the effects of GMFs on cell proliferation, expression levels of Akt protein, a protein kinase which can promote cell survival, were detected. Twenty-four hours after treatment with a GMF, the phosphoAkt/Akt ratio was significantly decreased in PC-3 cells in the GMF 10 minutes and GMF 20 minutes groups when compared with the $\mathrm{NC}$ group $(P<0.05$; Figure 3$)$, indicating that Akt signaling was inhibited under exposure of the GMF.

\section{Gyromagnetic fields induced cell apoptosis in PC-3 cells}

To investigate if GMFs have an impact on cell apoptosis in PC-3 cells, we elucidated the rate of cell apoptosis by performing flow cytometry assays. Twenty-four hours after the influence of a GMF, late apoptotic cell rate was significantly increased in the GMF groups $(P<0.05)$, while no significant differences were observed on the early apoptotic cell rate among groups ( $P>0.05$; Figure 4$)$. The data suggested that GMFs may have an effect of inducing cell apoptosis in PC-3 cells.

\section{Gyromagnetic fields activated p38 MAPK/ Caspase signaling in PC-3 cells}

To understand the mechanism of the effects of GMFs on cell apoptosis, expression levels of proteins in apoptosis associated p38 MAPK/Caspase signaling were detected. Twentyfour hours after GMF treatment, western blot analysis showed that phospho-p38 MAPK/p38 MAPK ratio and Cleaved Caspase-9 expression levels were significantly increased in PC-3 cells ( $P<0.05$; Figure 5A-C), and also immunofluorescence staining showed that Cleaved Caspase-3-positive cell numbers were significantly increased in the GMF 10-minute group ( $P<0.05$; Figure 5D and $\mathrm{E})$. These results indicated that p38 MAPK/Caspase signaling was activated under exposure to GMFs. 
A

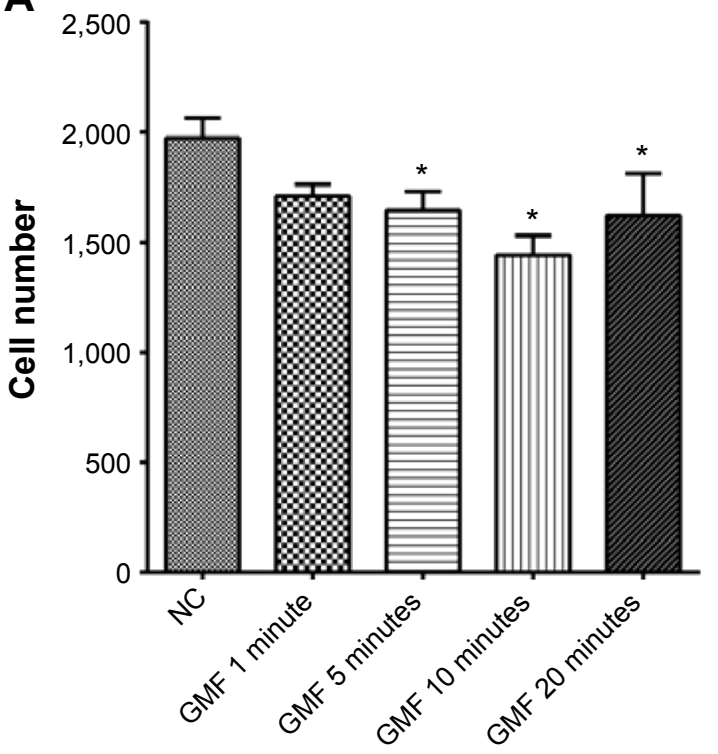

B

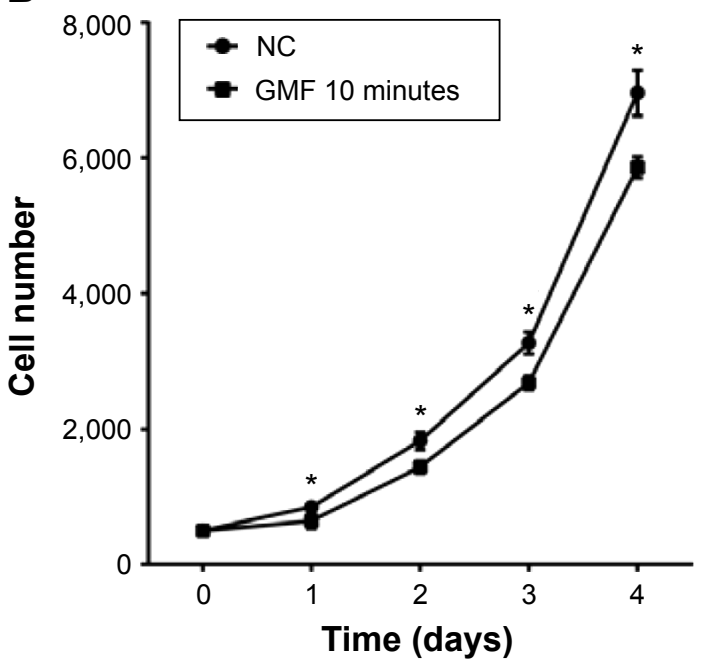

C
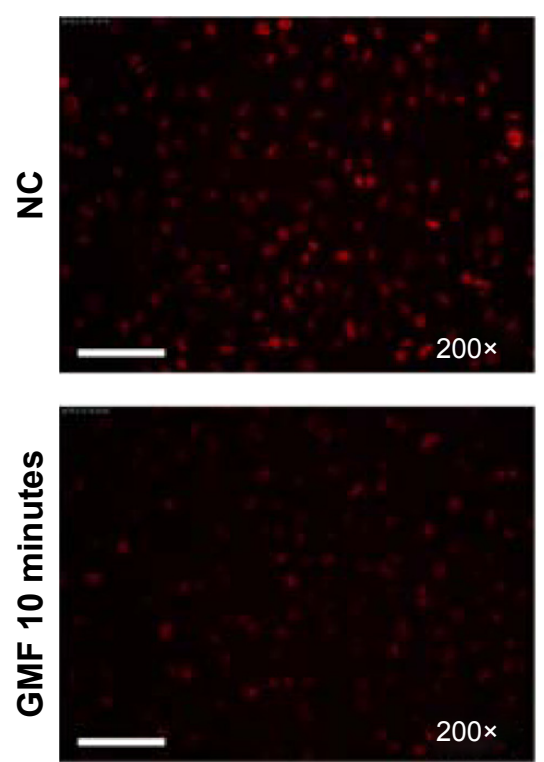

DAPI
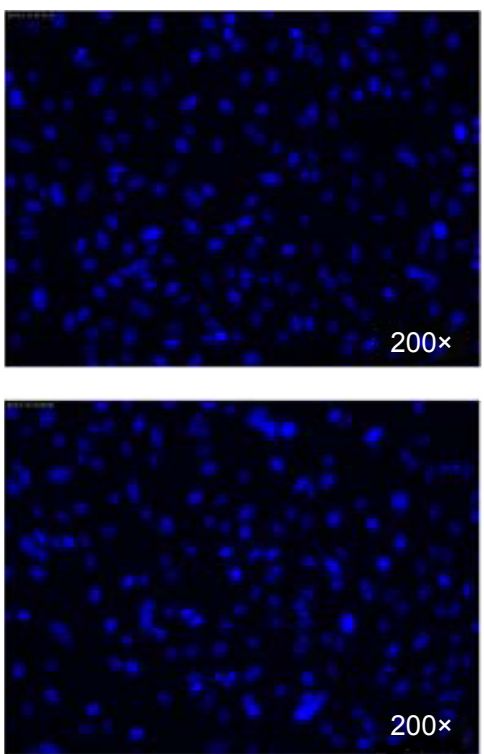

D

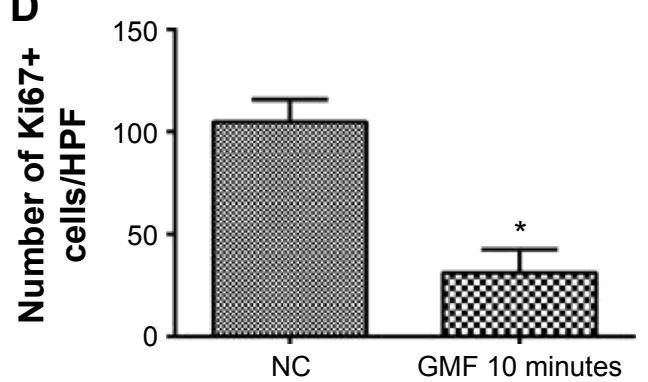

Figure 2 Effect of gyromagnetic fields on cell proliferation in PC-3 cells.

Notes: (A) Quantitative analysis of cell number counting assayed with CCK-8 in PC-3 cells after 24 hours treatment with a gyromagnetic field from the normal control (NC) group and the gyromagnetic field (GMF) groups with treatment times of I, 5, I0, and 20 minutes $(\mathrm{n}=3)$. (B) Cell numbers were checked 24 hours after each treatment with a gyromagnetic field in PC-3 cells from the NC group and cells treated with a magnetic field for 10 minutes each time (GMF 10 minutes, once a day); ( $n=3$ ). (C) Ki67 (red) immunofluorescence staining in PC-3 cells 24 hours after treatment with a gyromagnetic field from the NC group and cells treated once with a magnetic field for 10 minutes (GMF 10 minutes). Nuclei were labeled with 4',6-diamidino-2-phenylindole (DAPI) (blue). Scale bars=50 $\mu$ m, 200×. (D) Quantitative data of Ki67-positive cell number per high power field (HPF) by Image-Pro plus $(n=3)$. $* P<0.05$ when compared with the NC group. 
A

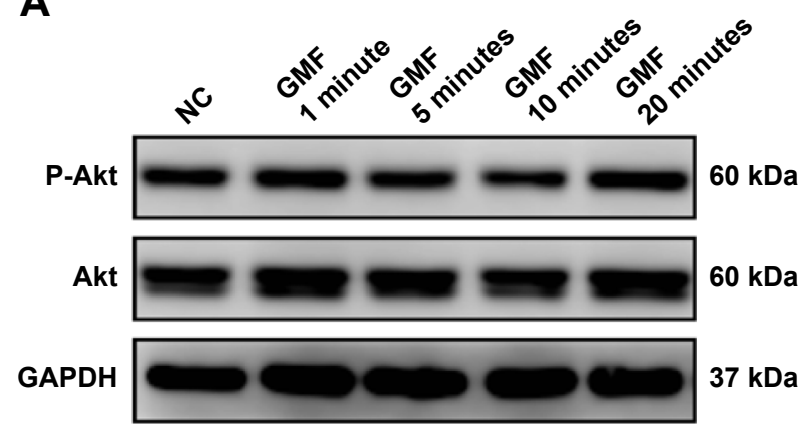

B

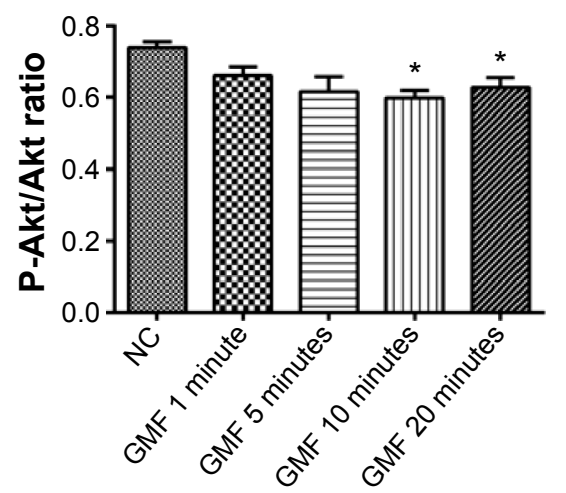

Figure 3 Effect of gyromagnetic fields on Akt signaling in PC-3 cells.

Notes: (A) Western blot analysis for phospho-Akt and Akt expression in PC-3 cells 24 hours after treatment with a gyromagnetic field from the normal control (NC) group and the gyromagnetic field (GMF) groups with treatment times of I, 5, 10, and 20 minutes. (B) Quantitative data of band intensity for phospho-Akt and Akt by ImageJ ( $\mathrm{n}=3$ ). $* P<0.05$ when compared with the NC group.

\section{Gyromagnetic fields regulated cell polarity conditions in PC-3 cells}

To demonstrate the effects of GMFs on cell polarity regulation, immunofluorescence staining of PARD3, a crucial regulator of cell polarization and tumorigenesis, was detected 24 hours after treatment with a GMF. PARD3-positive cell number was significantly increased in the GMF 10 minutes group when compared with the NC group $(P<0.05$; Figure 6). The data suggested that abnormal cell polarity conditions of tumor cells were regulated by GMFs in PC-3 cells.

\section{Discussion}

Surgery is now the most effective treatment for prostate cancer but several complications such as incontinence, hematuria, gastrointestinal toxicity, proctopathy, and erectile dysfunction have been reported. ${ }^{19}$ After the operation, patients experience extra suffering and so invasive surgery for prostate cancer treatment may not be ideal. For this reason, non-invasive methods without obvious side effects for treating prostate cancer are urgently needed. Magnetic field therapy belongs to the field of physical therapy and has been reported to play an important role in nerve regeneration, pain relief, and anti-inflammation. ${ }^{13,20,21}$ Furthermore, no such side effects have been revealed in the literature, making it a potential adjuvant therapy for surgical operations. Moreover, the PC-3 cell line was initiated from bone metastasis of a grade IV prostatic adenocarcinoma from a 62-year-old male Caucasian, which makes it a good model for metastatic androgen-independent prostate cancer research.

Magnetic field therapeutic modalities can be categorized into static magnetic fields and time-varying magnetic fields. ${ }^{22}$ Magnetic fields that are applied differ as their physical parameters change. The transformation progress of magnetic stimulation into bioeffects and the mechanism of biological response to such a form of physical therapy is still not completely understood. It is believed that there are several physical and biological effects caused by the following static magnetic fields: eddy currents by displacement, Lorentz force, magnetic force, magnetic torque, and radical pair effect. ${ }^{22}$ There are two types of mechanical effects that a static magnetic field can exert on biological bodies: magneto-orientation and magneto-mechanical translation. ${ }^{23}$ When it comes to timevarying magnetic fields, eddy currents, and thermal effects are observed. ${ }^{22}$ The frequency of time-varying magnetic fields ranges from $1 \mathrm{~Hz}$ to $100 \mathrm{kHz}$; thermal effects are too small to be considered. ${ }^{24}$ In the present study, the GMF outputted by a CKJ-II (QLX-II) gyromagnetic therapeutic machine belonged to the time-varying magnetic fields. It is believed that the interaction between GMFs and human prostatic cancer cells induces eddy currents within the cells, which may further affect cell proliferation and apoptosis. Moreover, the International Commission on Non-Ionizing Radiation Protection guidelines recommend that the magnetic flux density of reference levels for public exposure to time-varying magnetic fields is $40 \mathrm{mT} / \mathrm{Hz}^{2}$ for $1-8 \mathrm{~Hz}$ magnetic fields. ${ }^{24}$ The magnetic flux density of the GMF used in the present study $\left(8.3 \mathrm{mT} / \mathrm{Hz}^{2}\right)$ was below the limits in the International Commission on NonIonizing Radiation Protection guidelines, and this indicates that it is a safe method for public health.

Akt, also known as protein kinase B that was discovered as an oncogene, is a serine/threonine protein kinase that functions as a critical regulator of cell proliferation and survival; phosphorylated Akt exerts cellular functions in cell proliferation, survival, and differentiation. ${ }^{25}$ The significantly decreased 

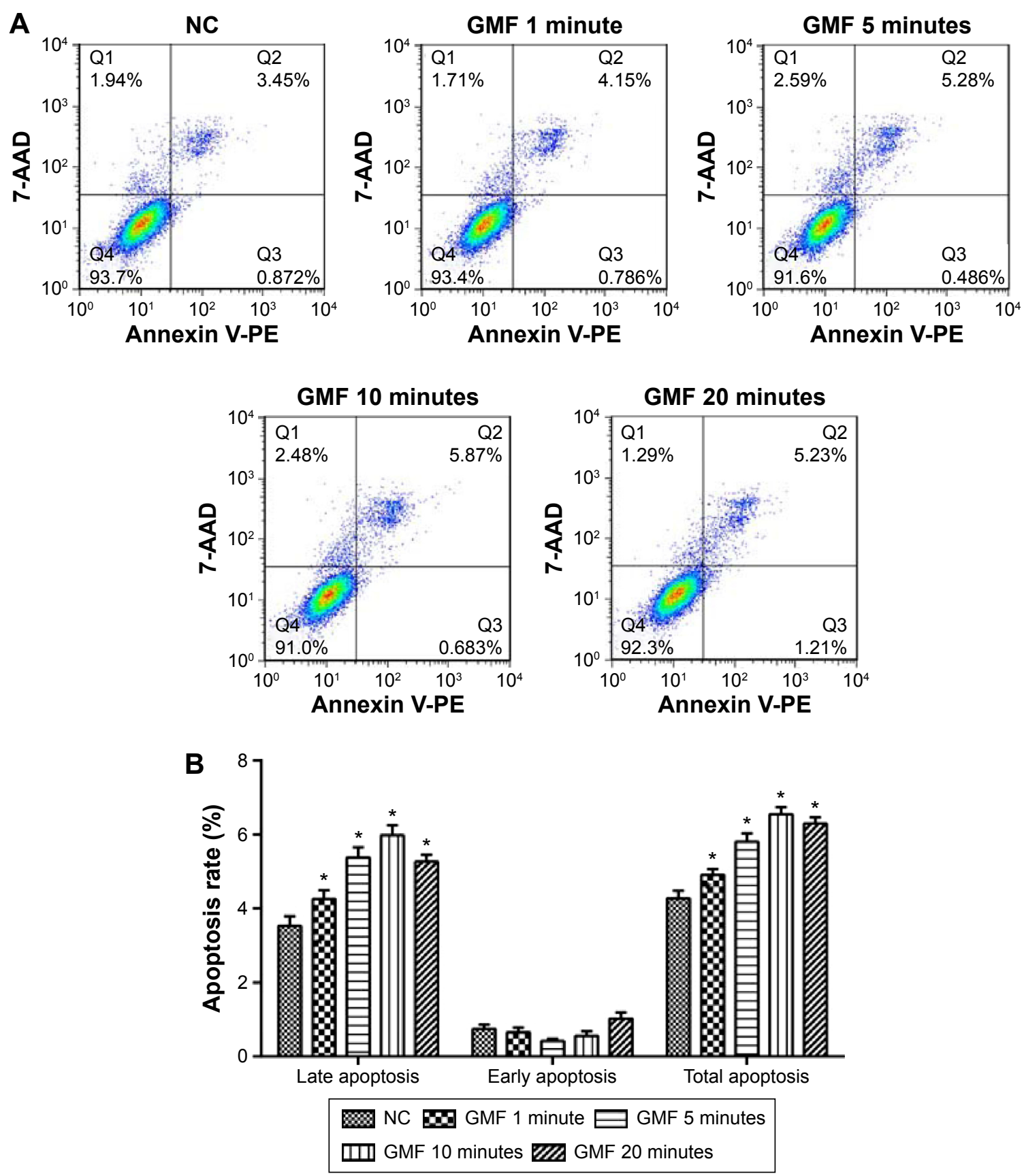

Figure 4 Effect of gyromagnetic fields on cell apoptosis in PC-3 cells.

Notes: (A) Flow cytometry analysis using an Annexin V-PE/7-AAD Apoptosis Detection Kit on PC-3 cells 24 hours after treatment with a gyromagnetic field from the normal control (NC group) and the gyromagnetic field (GMF) groups with treatment times of I, 5, 10, and 20 minutes. (B) Quantitative data of cell apoptosis rate by FlowJO $(n=3)$. Cells in late apoptosis were both Annexin V-PE positive and 7-AAD positive (Q2), and cells in early apoptosis were Annexin V-PE positive and 7-AAD negative (Q3). $* P<0.05$ when compared with the NC group.

phospho-Akt/Akt ratio under GMFs stimulation in the study may play an important role in inhibited cell proliferation and survival in PC-3 cells. On the other hand, p38 MAPKs are subfamilies of MAPKs, and MAPKs alter cell growth, proliferative potential, metabolism rate, and apoptosis when cells respond to stress or changes in physical and chemical properties of the environment. ${ }^{26}$ Caspases (cysteine-dependent aspartate-specific proteases) play important roles in regulating programmed cell death (or apoptosis) by performing selective, limited cleavage of key cellular signaling components. ${ }^{27}$ The activated p38 MAPK may regulate downstream protein Caspases to mediate its anti-proliferative functions. ${ }^{28}$ In the present study, the GMF was obviously a stress source for PC-3 cells, which may induce the activation of p38 MAPK. 

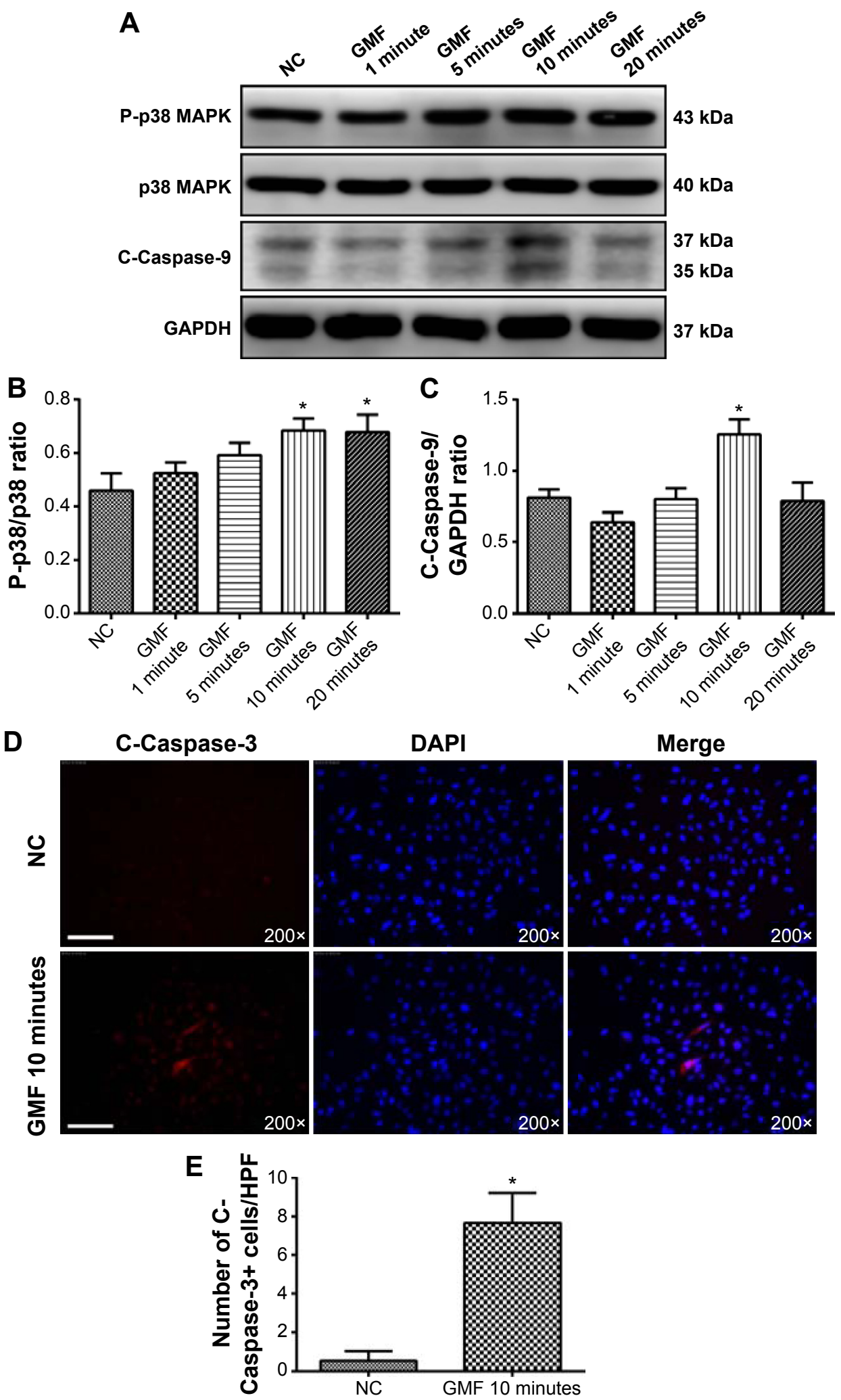

Figure 5 Effect of gyromagnetic fields on p38 MAPK/Caspase in PC-3 cells.

Notes: (A) Western blot analysis for phospho-p38 MAPK, P38 MAPK, and Cleaved Caspase- 9 expression in PC-3 cells 24 hours after treatment with a gyromagnetic field from the normal control (NC) group and the gyromagnetic field (GMF) groups with treatment times of I, 5, I0, and 20 minutes. (B and C) Quantitative data of band intensity for phospho-p38 MAPK, P38 MAPK, and Cleaved Caspase-9 by ImageJ ( $n=3$ ). (D) Cleaved Caspase-3 (red) immunofluorescence staining in PC-3 cells at 24 hours after treatment with a gyromagnetic field from the NC group and cells treated once with a magnetic field for 10 minutes (GMF 10 minutes). Nuclei were labeled with 4 ',6diamidino-2-phenylindole (DAPI) (blue). Scale bars $=50 \mu \mathrm{m}, 200 \times$. (E) Quantitative data of Cleaved Caspase-3-positive cell number per high power field (HPF) by Image-Pro plus $(n=3) . * p<0.05$ when compared with the NC group. 

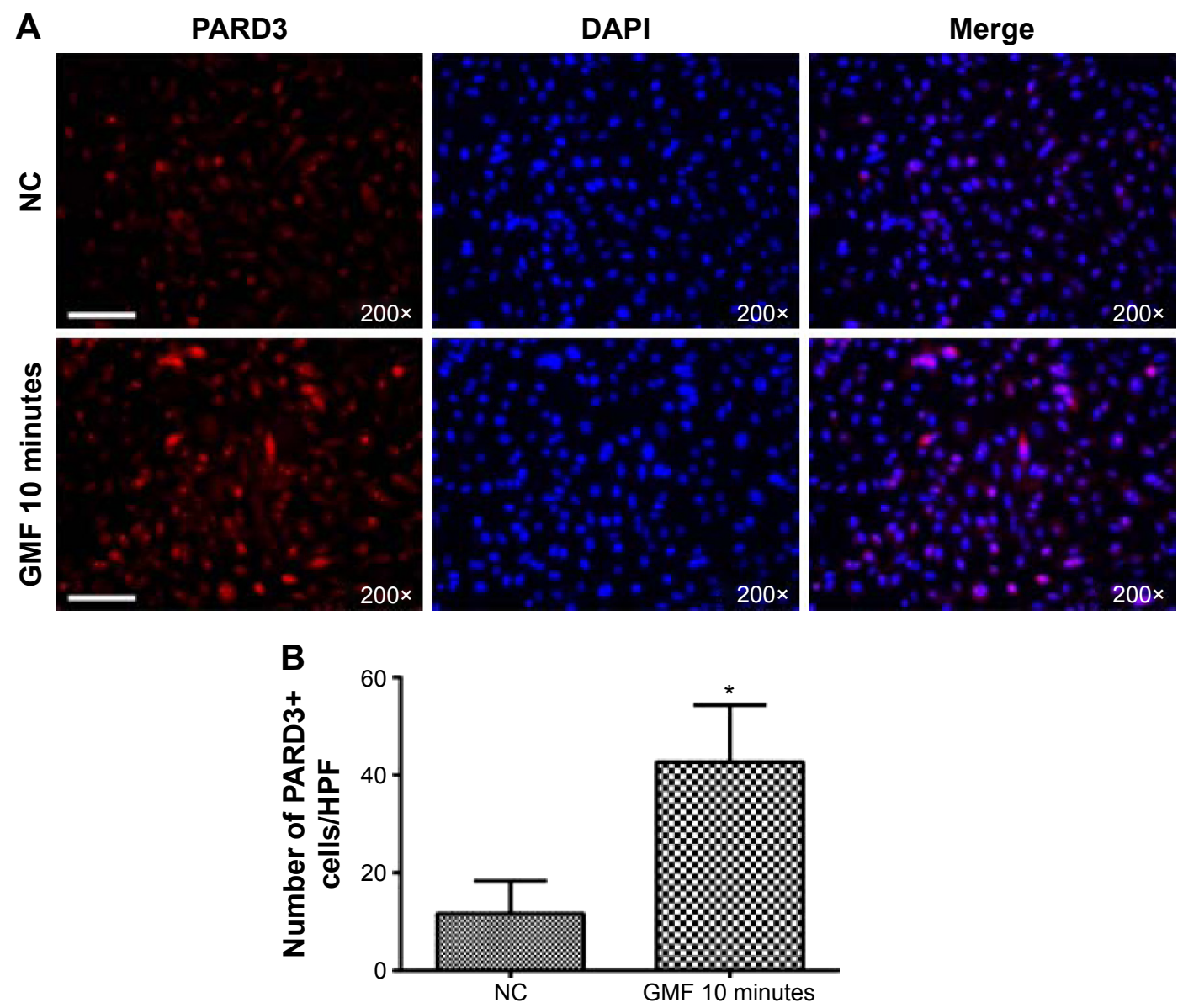

Figure 6 Effect of gyromagnetic fields on PARD3 protein in PC-3 cells.

Notes: (A) PARD3 (red) immunofluorescence staining in PC-3 cells 24 hours after treatment with a gyromagnetic field from the normal control (NC group) and cells treated once with a magnetic field for 10 minutes (GMF 10 minutes). Nuclei were labeled with 4',6-diamidino-2-phenylindole (DAPI) (blue). Scale bars =50 $\mu$ m, $200 \times$. (B) Quantitative data of PARD3-positive cell number per high power field (HPF) by Image-Pro plus ( $n=3)$. $* P<0.05$ when compared with the NC group.

Subsequently, the activated p38 MAPK regulated protein Caspase- 3 and protein Caspase-9 to exert apoptotic functions. The results indicate that GMFs may inhibit cell proliferation and induce apoptosis at least potentially through activation of the p38 MAPK/Caspase signaling pathway.

Polarization is crucial for normal cell physiology and tissue homeostasis. PARD3, a key element in the Par complex (a crucial regulator of apical-basal polarity, which includes PARD3, PARD6, aPKC, and small GTPases, such as $\mathrm{CDC} 42$ or RAC1), could promote junction assembly through regulation of actin dynamics, thus playing a crucial role in the formation and maintenance of cell apical-basal polarity. ${ }^{29}$ In addition, tight junctions could form an apicalbasal barrier that prevents paracellular leakage of fluids and intermixing of membrane proteins between the apical and basolateral surfaces. The transformations of loss of cell polarity and disruption of cell junctions are frequently observed in the process of tumorgenesis. ${ }^{30}$ Zen et $\mathrm{al}^{31} \mathrm{dem}-$ onstrated that the PARD3 gene is deleted in $15 \%$ of primary esophageal squamous cell carcinoma and that mRNA levels are downregulated in 23 of 33 esophageal squamous cell carcinoma tumor tissues compared to matched controls. In the present study, the GMF could up-regulate the expression of PARD3, indicating that the corrupted cell polarity and cell junctions in cancer cells may be repaired to some extent under the stimulation of a GMF.

However, before wide application in clinics, optimization of frequency, magnetic field strength, and the exposure cycle for GMFs therapy need to be further investigated.

\section{Conclusion}

GMFs could potentially inhibit cell proliferation, induce apoptosis, and regulate tumor cell polarity conditions through the downregulation of Akt signaling, upregulation of p38 MAPK/Caspase signaling, and promotion of PARD3 protein expression in human prostatic adenocarcinoma PC-3 cells. Our findings suggest that GMFs may be a promising noninvasive strategy for treating prostate cancer. 


\section{Acknowledgments}

This work is supported by the Specialized Research Fund for the Doctoral Program of Higher Education of China (No 20120001110021).

\section{Disclosure}

The authors report no conflicts of interest in this work.

\section{References}

1. Center MM, Jemal A, Lortet-Tieulent J, et al. International variation in prostate cancer incidence and mortality rates. Eur Urol. 2012;61(6): 1079-1092.

2. Hoffman RM. Clinical practice. Screening for prostate cancer. $N$ Engl J Med. 2011;365(21):2013-2019.

3. Vickers AJ, Ulmert D, Sjoberg DD, et al. Strategy for detection of prostate cancer based on relation between prostate specific antigen at age $40-55$ and long term risk of metastasis: case-control study. $B M J$. 2013;346:f2023.

4. Ilic D, Neuberger MM, Djulbegovic M, Dahm P. Screening for prostate cancer. Cochrane Database Syst Rev. 2013;1:CD004720.

5. Damber JE, Aus G. Prostate cancer. Lancet. 2008;371(9625):1710-1721.

6. Droz JP, Aapro M, Balducci L, et al. Management of prostate cancer in older patients: updated recommendations of a working group of the International Society of Geriatric Oncology. Lancet Oncol. 2014;15(9): e404-e414.

7. Williams S, Chiong E, Lojanapiwat B, Umbas R, Akaza H; Asian Oncology Summit 2013. Management of prostate cancer in Asia: resource-stratified guidelines from the Asian Oncology Summit 2013. Lancet Oncol. 2013;14(12):e524-e534.

8. Shore ND, Karsh L, Gomella LG, Keane TE, Concepcion RS, Crawford ED. Avoiding obsolescence in advanced prostate cancer management: a guide for urologists. BJU Int. 2015;115(2):188-197.

9. Kafatos FC. A revolutionary landscape: the restructuring of biology and its convergence with medicine. J Mol Biol. 2002;319(4):861-867.

10. Leoci R, Aiudi G, Silvestre F, Lissner E, Lacalandra GM. Effect of pulsed electromagnetic field therapy on prostate volume and vascularity in the treatment of benign prostatic hyperplasia: a pilot study in a canine model. Prostate. 2014;74(11):1132-1141.

11. Giannakopoulos XK, Giotis C, Karkabounas S, et al. Effects of pulsed electromagnetic fields on benign prostate hyperplasia. Int Urol Nephrol. 2011;43(4):955-960.

12. Sadeghipour R, Ahmadian S, Bolouri B, Pazhang Y, Shafiezadeh M. Effects of extremely low-frequency pulsed electromagnetic fields on morphological and biochemical properties of human breast carcinoma cells (T47D). Electromagn Biol Med. 2012;31(4):425-435.

13. Suszynski K, Marcol W, Szajkowski S, et al. Variable spatial magnetic field influences peripheral nerves regeneration in rats. Electromagn Biol Med. 2014;33(3):198-205.

14. Kanat E, Alp A, Yurtkuran M. Magnetotherapy in hand osteoarthritis: a pilot trial. Complementary Therapies Med. 2013;21(6): 603-608.
15. Horvath K, Kulisch A, Nemeth A, Bender T. Evaluation of the effect of balneotherapy in patients with osteoarthritis of the hands: a randomized controlled single-blind follow-up study. Clin Rehabil. 2012;26(5): 431-441.

16. Ding S, Peng H, Fang HS, Zhou JL, Wang Z. Pulsed electromagnetic fields stimulation prevents steroid-induced osteonecrosis in rats. $B M C$ Musculoskelet Disord. 2011;12:215.

17. Plank C, Zelphati O, Mykhaylyk O. Magnetically enhanced nucleic acid delivery. Ten years of magnetofection-progress and prospects. Adv Drug Deliv Rev. 2011;63(14-15):1300-1331.

18. Markov MS. Magnetic field therapy: a review. Electromagn Biol Med. 2007;26(1):1-23.

19. Tewari A, Sooriakumaran P, Bloch DA, Seshadri-Kreaden U, Hebert AE, Wiklund P. Positive surgical margin and perioperative complication rates of primary surgical treatments for prostate cancer: a systematic review and meta-analysis comparing retropubic, laparoscopic, and robotic prostatectomy. Eur Urol. 2012;62(1):1-15.

20. Rowe E, Smith C, Laverick L, Elkabir J, Witherow RO, Patel A A prospective, randomized, placebo controlled, double-blind study of pelvic electromagnetic therapy for the treatment of chronic pelvic pain syndrome with 1 year of followup. J Urol. 2005;173(6):2044-2047.

21. Gomez-Ochoa I, Gomez-Ochoa P, Gomez-Casal F, Cativiela E, Larrad-Mur L. Pulsed electromagnetic fields decrease proinflammatory cytokine secretion (IL-1beta and TNF-alpha) on human fibroblast-like cell culture. Rheumatol Int. 2011;31(10):1283-1289.

22. Yamaguchi-Sekino S, Sekino M, Ueno S. Biological effects of electromagnetic fields and recently updated safety guidelines for strong static magnetic fields. Magn Resonance Med Sci. 2011;10(1):1-10.

23. International Commission on Non-Ionizing Radiation Protection Guidelines on limits of exposure to static magnetic fields. Health Phys. 2009;96(4):504-514.

24. International Commission on Non-Ionizing Radiation Protection Guidelines for limiting exposure to time-varying electric and magnetic fields (1 Hz to $100 \mathrm{kHz}$ ). Health Phys. 2010;99(6):818-836.

25. Cheng JQ, Lindsley CW, Cheng GZ, Yang H, Nicosia SV. The Akt/ PKB pathway: molecular target for cancer drug discovery. Oncogene. 2005;24(50):7482-7492.

26. Cuenda A, Rousseau S. p38 MAP-kinases pathway regulation, function and role in human diseases. Biochim Biophys Acta. 2007;1773(8):1358-1375.

27. Li J, Yuan J. Caspases in apoptosis and beyond. Oncogene. 2008;27(48): 6194-6206.

28. Shimizu T, Nakazato T, Xian MJ, Sagawa M, Ikeda Y, Kizaki M. Resveratrol induces apoptosis of human malignant $\mathrm{B}$ cells by activation of caspase-3 and p38 MAP kinase pathways. Biochem Pharmacol. 2006 71(6):742-750.

29. Aranda V, Nolan ME, Muthuswamy SK. Par complex in cancer: a regulator of normal cell polarity joins the dark side. Oncogene. 2008;27(55) 6878-6887.

30. Huang L, Muthuswamy SK. Polarity protein alterations in carcinoma: a focus on emerging roles for polarity regulators. Curr Opin Genet Dev 2010;20(1):41-50.

31. Zen K, Yasui K, Gen Y, et al. Defective expression of polarity protein PAR-3 gene (PARD3) in esophageal squamous cell carcinoma. Oncogene. 2009;28(32):2910-2918.
OncoTargets and Therapy

\section{Publish your work in this journal}

OncoTargets and Therapy is an international, peer-reviewed, open access journal focusing on the pathological basis of all cancers, potential targets for therapy and treatment protocols employed to improve the management of cancer patients. The journal also focuses on the impact of management programs and new therapeutic agents and protocols on

\section{Dovepress}

patient perspectives such as quality of life, adherence and satisfaction. The manuscript management system is completely online and includes a very quick and fair peer-review system, which is all easy to use. Visit http://www.dovepress.com/testimonials.php to read real quotes from published authors. 